\title{
Valproic Acid-Induced Changes of 4D Nuclear Morphology in Astrocyte Cells
}

Alexandr A. Kalinin ${ }^{a, b, c}$, Xinhai Hou ${ }^{a, b, d}$, Alex S. Ade ${ }^{b}$, Gordon-Victor Fon ${ }^{b}$, Walter Meixner ${ }^{b}$, Gerald A. Higgins ${ }^{b}$, Jonathan Z. Sexton ${ }^{\mathrm{e}, \mathrm{f}, \mathrm{g}}$, Xiang Wan ${ }^{\mathrm{a}}$, Ivo D. Dinov ${ }^{\mathrm{b}, \mathrm{c}, \mathrm{h}}$, Matthew J. O'Meara ${ }^{\mathrm{b}}$, Brian D. Athey $\mathrm{b}^{\mathrm{b}, \mathrm{h}, \mathrm{i}}$ *

aShenzhen Research Institute of Big Data, Shenzhen 518172, Guangdong, China

${ }^{b}$ Department of Computational Medicine and Bioinformatics, University of Michigan, Ann Arbor, MI 48109, USA

'Statistics Online Computational Resource (SOCR), Health Behavior and Biological Sciences, University of Michigan, Ann Arbor, MI 48109, USA

${ }^{\mathrm{d} S}$ chool of Science and Engineering, Chinese University of Hong Kong-Shenzhen, Shenzhen 518172, Guangdong, China

eDepartment of Internal Medicine, Gastroenterology, Michigan Medicine at the University of Michigan, Ann Arbor, MI 48109, USA

fDepartment of Medicinal Chemistry, College of Pharmacy, University of Michigan, Ann Arbor, MI 48109, USA

${ }^{9}$ Center for Drug Repurposing, University of Michigan, Ann Arbor, MI 48109, USA

hMichigan Institute for Data Science (MIDAS), University of Michigan, Ann Arbor, MI 48109, USA

'Department of Psychiatry, University of Michigan, Ann Arbor, MI 48109, USA

${ }^{*}$ Corresponding author: bleu@umich.edu 


\begin{abstract}
Histone deacetylase inhibitors, such as valproic acid (VPA), have important clinical therapeutic and cellular reprogramming applications. They induce chromatin re-organization that is associated with altered cellular morphology. However, there is a lack of comprehensive characterization of VPA-induced changes of nuclear size and shape. Here, we quantify 3D nuclear morphology of primary human astrocyte cells treated with VPA over time (hence, 4D). We compared volumetric and surface-based representations and identified seven features that jointly discriminate between normal and treated cells with $85 \%$ accuracy on day 7 . From day 3 , treated nuclei were more elongated and flattened and then continued to morphologically diverge from controls over time, becoming larger and more irregular. On day 7 , most of the size and shape descriptors demonstrated significant differences between treated and untreated cells, including a $24 \%$ increase in volume and $6 \%$ reduction in extent (shape regularity) for treated nuclei. Overall, we show that 4D morphometry can capture how chromatin re-organization modulates the size and shape of the nucleus over time. These nuclear structural alterations may serve as a biomarker for histone (de-)acetylation events and provide insights into mechanisms of astrocytes-to-neurons reprogramming.
\end{abstract}

\title{
Introduction
}

Multi-cellular organisms regulate their cell type and state by selectively exposing portions of their genome for transcription through the spatial and temporal organization of chromatin-dubbed the 4D Nucleome (Chen et al., 2015; Cremer et al., 2015; Higgins et al., 2015, 2017a). Structurally, 3D conformation of the genome involves 1.65 turns of DNA wrapped onto a histone octamer, creating nucleosomes linked as beads-on-a-string, which are then wrapped into chromatin fibers and higher-order loops, organizing into topologically associating domains (TADs). Finally, TADs collect into a diploid set of chromosome territories (Higgins et al., 2015, 2017a). Histones accommodate a range of post-translational modifications, which are controlled by epigenetic proteins that ultimately regulate the transcriptional state of the cell and mediate mechanical protection of genome by chromatin rigidity. (Yang and Seto, 2007; Stephens et al., 2019). Chemicals that target these proteins can be used to modulate chromatin states and the concomitant cell and nuclear morphology changes observed in human diseases (Marchion et al., 2005; Stephens et al., 2019). For example, valproic acid (VPA) is a histone deacetylase inhibitor used clinically to treat epilepsy, bipolar disorders, social phobias, and neuropathic pain (Göttlicher et al., 2001; Ganai et al., 2015). Mechanistically, VPA shifts the balance towards greater histone acetylation, DNA exposure, and chromatin decondensation; activating transcriptional programs 
that regulate cellular processes related to cancer (Göttlicher et al., 2001; Eckschlager et al., 2017), traumatic brain injury (Higgins et al., 2017b), ischemia (Pickell et al., 2020), as well as cellular reprogramming (Huangfu et al., 2008). An outstanding question, however, is to characterize how mesoscale nuclear structures such as TADs mediate effects of VPA and other epigenetic modulators on cellular morphology. However, limitations of the emerging technologies that can capture these nano- (histone marks) and meso- (nuclear architecture) scale phenomena, including optical microscopy, ATACseq, and single cell RNAseq, make it difficult to simultaneously monitor macro-scale (cellular morphology) cellular state.

Recent mechanobiology studies revealed the role of chromatin as a key regulator of nuclear shape (Uhler and Shivashankar, 2018; Stephens et al., 2019). VPA induces increased euchromatin, which results in weakened nuclear rigidity and morphology changes that occur independently of lamins (Stephens et al., 2017, 2018). Here we aim to leverage this effect for assessing how the epigenetic and mesoscale nuclear state can be characterized by the overall nuclear size and shape to probe VPA's mechanism of action. Importantly, we show that nuclear morphology can be captured in standard microscopy experiments to relate epigenetic regulators with phenotypic measurements of a cell and its organelles.

Towards this goal, we present a detailed characterization of the size and shape of astrocyte nuclei in the context of VPA treatment. We chose astrocytes because they developmentally originate from the same precursor cells as neurons and proliferate in response to brain damage (Amamoto and Arlotta, 2014), but also can be directly reprogrammed into functional neurons by sets of small molecules_often including VPA-in the lab (Cheng et al., 2015; Zhang et al., 2015; Gao et al., 2017; Qin et al., 2017). These studies, however, are not always congruent. Some reports demonstrated that VPA inhibits (Yin et al., 2019) or increases reprogramming efficiency (Cheng et al., 2015; Zhang et al., 2015), while others suggest that VPA alone induces astrocyte reprogramming into neurons (Cheng et al., 2015). The treatment protocols, concentrations, and combinations of small molecules differ between these studies, indicating that underlying mechanisms driving the trans-differentiation process are not well understood.

Existing studies that have focused on astrocyte-to-neuron reprogramming observed changes only in cellular morphology, presented in a qualitative fashion. Others quantified VPA-induced nuclear morphological changes in non-astrocyte cells, reporting time-, dose-, and cell type-dependent response. Their results ranged from no observed changes in nuclear geometry (Ganai et al., 
2015); to altered nuclear shape ("blebbing"), but not size (Stephens et al., 2017, 2018); to increased nuclear size, either after 1 hour (Felisbino et al., 2011, 2014, 2016) or not earlier than after 7-14 days (Kortenhorst et al., 2009) of VPA treatment. Moreover, these approaches were limited to basic 2D surrogates of geometric measures, often reporting few features such as crosssectional nuclear area or maximum diameter. Given that chromatin is highly organized in 3D, the effects of chromatin remodeling on nuclear morphology can only be fully described in three dimensions. Supporting this assumption, there is growing practical evidence that 3D representations allow for more accurate characterizations of cell and nuclear morphology, compared to 2D measures (Choi and Choi, 2007; Meyer et al., 2009; Depeursinge et al., 2014; Kalinin et al., 2018a; Medyukhina et al., 2020). Since single-perspective 2D images depend on object's orientation and the focal plane, they provide only a sample of its real geometry. For example, cell nuclei that differ in volume and shape can appear similarly small and circular in optically sectioned images, thus leading to lower discriminative performance of a classifier (Choi and Choi, 2007). Besides, more comprehensive morphological representation of nuclei in 3D is more informative than resolution as a determining factor for classification performance (Meyer et al., 2009). Our previous results also indicated that 3D size and shape descriptors outperform their 2D counterparts in the task of nuclear morphological classification (Kalinin et al., 2018a).

Even when the 3D object is perfectly aligned with the focal plane, only the first two out of three principal axes (major, median, minor) can be measured from its $2 \mathrm{D}$ representation. This reduces accuracy of shape measures computed from size features. For example, sphericity assesses the compactness of the object, i.e., it measures how closely the global object shape resembles that of a perfect sphere, which is computed via volume and surface area or approximated using principal axes (Xu et al., 2009). Extent (ratio of the object volume to the bounding box volume) and solidity (ratio of the object volume to the convex hull volume) of nuclear surfaces are useful measures of the amount and size of concavities (or protrusions) in an object boundary. Other shape descriptors rely on the notion of the curvature that describes how bent the curve is around each point of a surface. They allow for measuring local shape alterations that are observed on the nuclear surface and would not be exhibited in 2D projection. Mean curvature is an extrinsic measure of $3 \mathrm{D}$ shape that provides a balanced measure between shape morphology and curvature magnitude (Tsagkrasoulis et al., 2017; Kalinin et al., 2018b). Gaussian curvature is an intrinsic (scale-invariant) measure of curvature that depends only on distances that are measured on the surface. Shape index and curvedness are morphometric descriptors that can capture local shape features, independently or in relation to the size of an object (Koenderink and Van Doorn, 
1992). Fractal dimension is the measure of the object's boundary complexity (Metze et al., 2019). Together, these features allow one to measure various aspects of shape and provide a detailed quantitative characterization of 3D object morphology.

To address the limitations of previous studies, we quantified VPA-induced changes in 4D nuclear morphology of primary human astrocyte cells. Our findings show that geometric descriptors extracted from voxel and surface-modeled representations of 3D nuclear shapes enabled accurate and interpretable characterization of time-dependent morphological changes in VPAtreated astrocytes. This allowed us to distinguish between nuclear morphological profiles of treated and normal astrocytes over time with a time-average accuracy of $82 \%$. We showed that VPA treatment induced a time-dependent increase in nuclear size and nuclear shape irregularity in astrocytes over the course of treatment.

\section{Results and Discussion}

\section{Experiment and data}

In order to determine how VPA-induced alterations of chromatin structure are reflected in 4D nuclear morphology, we treated human astrocyte cells with $1.5 \mathrm{mM}$ of VPA at multiple time points (days 1, 3, and 5) and obtained volumetric images of DAPI-labeled nuclei using confocal microscopy at 3 time points (days 3, 5, and 7). This provided us with 4D images (3D+time) in following conditions: normal human astrocytes (NHA) and cells treated with VPA (VPA) (Figure 1A). First, we used deconvolution to correct for background noise and spherical aberrations in original image volumes (Supplementary Figure 1). Then, we segmented individual nuclei into 3D binary voxel masks (Figure 1B). The number of nuclei identified after segmentation and quality control are listed in Table 1 for each day and treatment condition. Higher numbers of untreated nuclei compared to the treated group might be due to significant inhibition of astrocyte cell growth by VPA at concentrations over $1 \mathrm{mM}$ (Sasai et al., 2007). Details of the deconvolution, segmentation, and quality control protocols can be found in the Methods section.

Table 1. Number of segmented astrocyte nuclear 3D binary masks per day for each treatment condition after QC (number of nuclei filtered by quality control is in parenthesis).

\begin{tabular}{lccc}
\hline & \multicolumn{3}{c}{ Day } \\
\cline { 2 - 4 } Treatment & $\mathbf{3}$ & $\mathbf{5}$ & $\mathbf{7}$ \\
\hline NHA & $186(-23)$ & $163(-18)$ & $101(-10)$ \\
\hline
\end{tabular}




\begin{tabular}{lccc}
\hline VPA & $128(-18)$ & $67(-6)$ & $78(-15)$ \\
\hline Total & $314(-41)$ & $230(-24)$ & $179(-35)$ \\
\hline
\end{tabular}

A simple approach to 3D morphometry is to extract features from binary object masks represented as voxel volumes (Dufour et al., 2015). From each voxel binary mask (Figure 1B), we computed the total of 11 geometric features: volume, bounding box and convex hull volumes, extent, solidity, lengths of main axes, and inertia tensor principal components. However, voxel-based representations can be noisy and may lose fine local geometric detail or even misrepresent the object's global topological structure. We have previously shown that nuclear surfaces obtained via shape modeling are more informative and reliable for nuclear morphometry when compared to alternatives (Kalinin et al., 2018b). Here, we extended that approach by extracting 16 different size and shape descriptors that can characterize morphological changes in more detail, compared to just 6 in our previous study (Kalinin et al., 2018b). We obtained nuclear surface representations from 3D binary masks (Figure 1B) and measured the same descriptors as extracted from voxels, with the addition of bounding cylinder and sphere volumes, sphericity, fractal dimension, mean and Gaussian curvatures, curvedness, and shape index. The combined feature tensor containing nuclear morphological profiles (Figure 1B) was used for model and feature selection, statistical and machine learning-based analysis, and interpretation (Figure 1C).

\section{Surface modeling provided compact and accurate characterization of 3D nuclear morphology}

To evaluate the utility of different representations of nuclear morphology, we asked how well they facilitate morphological classification of NHA vs VPA cells, using timepoint-averaged area under the receiving operator characteristic curve $(A \cup C)$ as the performance metric. Since performance of classification algorithms varies across bioinformatics problems and datasets (Olson et al., 2018) and not all models have feature weighting mechanism, we compared the performance across eight standard classifiers that enable feature importance estimation (Supplementary Figure 2). At each timepoint, we used random subsampling of the prevalent class due to high class imbalance (Table 1). First, since voxel and surface features have not been directly compared before in terms of nuclear morphological classification, we trained each model on sets of voxel-based $(V)$ and surface-based features $(S)$. As a baseline, we defined 2D voxel features extracted from 3D binary mask maximum intensity projections $\left(V_{2 d}\right)$. To facilitate a fairer comparison between $V$ and $S$, we also evaluated the subsets $V_{\text {sub }}$ and $S_{\text {sub }}$ that consisted of the 10 features that are captured by both representations (Supplementary Figure 2). As expected, 
the $2 \mathrm{D}$ feature set performed the worst (73\% AUC), confirming that 3D measures provide more discriminative power. The performance was higher on the subset of voxel features $(77 \%$ vs $74 \%$ AUC on $V_{\text {sub }}$ vs $S_{\text {sub }}$ ), but not with full sets (77\% AUC on $V$ and on $S$ ). The best overall performance was achieved when using the combination of both voxel and surface-based features (78\% AUC on $V+S$ ). Among tested classifiers, the support vector machine (SVM) model (Cortes and Vapnik, $1995)$ outperformed other classifiers averaged across all feature sets (81\% AUC), so we chose to use this model going forwards.

Clustering of the complete $V+S$ feature set revealed 2 major groups, roughly corresponding to size and shape descriptors (Figure 2A). To reduce redundancy and aid interpretability, we selected seven features $\left(S_{7}\right)$ from smaller sub-clusters that maximized SVM classification performance ( $82 \%$ AUC). These features included surface-based median axis length, convex hull volume, bounding sphere volume, sphericity, average mean curvature, shape index, and voxelbased solidity. $S_{7}$ provided distinctive representations of nuclear morphological profiles at different time points, as shown by the t-SNE (van der Maaten and Hinton, 2008) 2D projection in Figure 2B. The SVM classifier demonstrated robust performance with AUCs of $80 \%, 80 \%$, and $85 \%$ on days 3,5 , and 7 , respectively (Figure $2 \mathrm{C}$ ), with classification errors that were only slightly shifted towards false negatives at each day, indicating the effectiveness of the prevalent class subsampling (Figure 2D). Relative feature ranking from the trained SVM model (Figure 2E) revealed that sphericity was the most important feature for day 3 , followed by convex hull and bounding sphere volumes. On day 5 , bounding sphere volume, sphericity, and solidity were the top-3 important descriptors. Bounding sphere volume was again the most important measure on day 7 , followed by average mean curvature, median axis length, and sphericity. Our findings showed that both size and shape features were important for discrimination of treated and untreated nuclei, demonstrating that a combination of $3 \mathrm{D}$ descriptors aids in accurate morphological classification.

\section{VPA induced increased nuclear size}

We observed time-dependent alterations in astrocyte nuclear sizes as demonstrated by examples of NHA and VPA-treated reconstructed nuclear surfaces shown in Figure 3A, with the latter having increasingly longer major axis length and higher volume. To provide a more detailed characterization of changes in 3D nuclear size, we chose six size descriptors for further study; three of which were selected as a part of $S_{7}$ (median axis length, convex hull, and bounding sphere volumes), while major and minor axes lengths and nuclear volume were chosen manually 
for interpretability (Figure 3B). Measuring the three principal axes also allowed for the generation of inferences about the global shape of nuclei, as described in the next section. We reported each relative difference as the percentage change from the mean of the control group (NHA), along with a $p$-value obtained using two-sided Mann-Whitney $U$ test with Holm-Šidák multiple testing correction, and AUC that in the case of Mann-Whitney $U$ test is equivalent to the common language effect size statistic (Mason and Graham, 2002).

By day 3, the average major axis length of VPA treated nuclei compared to controls had increased by $10 \%$ ( $p<0.0001,70 \%$ AUC), the average minor axes length had decreased by $7 \%(p<0.0001$, $67 \%$ AUC), while there was no significant difference in median axis lengths (Figure 3B). Despite more subtle changes in nuclear $(+5 \%, p<0.05,58 \%$ AUC) and convex hull volumes $(+4 \%, p>0.05$, $57 \%$ AUC), the volume of the bounding sphere was $36 \%$ larger $(p<0.0001,74 \%$ AUC) in the VPA group. Between days 3 and 5, measures of major and median axes lengths indicated intra-group changes for both NHA $(+12 \%, p<0.0001$ for major; $+13 \%, p<0.0001$ for median) and VPA nuclei $(+14 \%, p<0.0001$ for major; $+13 \%, p<0.001$ for median), along with slight shortening of minor axes. Nuclear, convex hull and bounding sphere volumes also increased in both groups, correspondingly. As the result, treated nuclei differed from controls on day 5 by having $13 \%$ longer major axis ( $p<0.0001,73 \%$ AUC) and $4 \%$ shorter minor axis ( $p<0.0001,67 \%$ AUC), along with $48 \%$ larger bounding sphere volumes ( $p<0.0001,74 \%$ AUC).

Between days 5 and 7, there were no significant changes in axes lengths in the control group, while median axis length in treated group increased by $13 \%(p<0.001)$ and minor increased by $5 \%(p<0.05)$ (Figure 3B). By day 7, VPA-treated nuclei had 15\% larger major axes $(p<0.0001$, $75 \%$ AUC) and $15 \%$ larger median axes ( $p<0.0001,70 \%$ AUC), while minor axis length was comparable to that of untreated nuclei. Nuclear volume and convex hull volume increased in both groups from day 5 to 7 , but the change in VPA-treated nuclei was more dramatic $(+23 \%, p<0.0001$ for volume; $+22 \%, p<0.0001$ for convex hull) than in the NHA group $(+8 \%, p<0.001$ for volume; $+7 \%, p<0.01$ for convex hull). As a result, the treated nuclei had $24 \%$ larger volume $(p<0.0001$, $77 \%$ AUC), $25 \%$ larger convex hull ( $p<0.0001,76 \%$ ), and $64 \%$ larger bounding sphere volume $(p<0.0001,80 \%$ AUC), when compared to controls on day 7 .

Our findings highlight a transition point between two phases of morphological changes. Before day 5, nuclear sizes in both VPA and NHA groups significantly increased in all reported measures, except for the minor axis length. VPA-treated nuclei demonstrated more prominent elongation 
and flattening, along with the slightly more rapid increase of the volume, compared to controls. NHA nuclear volumes increased less between days 5 and 7, while measures of VPA-treated nuclear size continued to increase, mostly due to longer median axes, demonstrating biggest differences by the last day, as illustrated by visualizations in Figure 3A. Finally, all individual feature AUCs were lower than those of the SVM model at each timepoint (Figure 2C), which highlighted the ability of a combination of $3 D$ linear and volumetric features to capture size alterations that are difficult to ascertain when using solely individual measures.

\section{VPA induced nuclear shape irregularity}

Consistent with VPA decondensing the chromatin to reduce nuclear rigidity (Stephens et al., 2018), we observed more globally irregular surface shapes (Figure 4 A and C) and occasional blebbing (Figure 4C). As reported by the size measures (Figure 3B), VPA induced nuclear elongation and flattening that were reflected in more ellipsoidal and less spherical shape. To quantify, by day 3, VPA-treated nuclei demonstrated lower sphericity $(-3 \%, p<0.0001,72 \%$ AUC) and higher average mean curvature $(+3 \%, p<0.0001,71 \% \mathrm{AUC})$, compared to the NHA group (Figure 4B), which indicated the presence of more convex and less concave points on the surface (Figure 4A). Average Gaussian curvature and curvedness of VPA-treated nuclei were slightly lower $(-5 \%, p<0.05,58 \%$ AUC for Gaussian curvature; $-5 \%, p<0.01,59 \%$ AUC for curvedness) than those of controls, which also corresponded to overall less spherical objects. Between days 3 and 5 , there were similar intra-group decreases in sphericity, shape index, and solidity that corresponded to increase in size of both treated and untreated nuclei at the same timepoint. More subtle alterations also included decrease in average Gaussian curvature in both NHA $(-1 \%$, $p<0.05)$ and VPA $(-5 \%, p<0.05)$ groups, along with the decrease of fractal dimension of the untreated nuclei $(-0.2 \%, p<0.01)$ (Figure 4B). Differences between shape descriptors extracted from two conditions on day 5 were similar to those from day 3 . While the difference in solidity and shape index were not identified as 'statistically significant' at the chosen $p$-value cut offs, they contributed to discriminative ability of the SVM classifier on both days 3 and 5 (Figure 2D), indicating their importance.

By day 7, NHA nuclei only demonstrated a $2 \%$ decrease in average mean curvature $(p<0.05)$ from the previous timepoint across all shape features. The VPA group exhibited an $11 \%$ decrease in curvedness $(p<0.0001)$ and a $7 \%$ decrease in average Gaussian curvature $(p<0.01)$ (Figure 4B). As on days 3 and 5, treated nuclei were still less spherical and had higher average mean curvature on day 7 compared to controls. However, decreases in curvedness $(-15 \%, p<0.0001$, 
$74 \%$ AUC) and average Gaussian curvature $(-10 \%, p<0.0001,71 \%$ AUC) were also more prominent at the last timepoint. Moreover, VPA-treated nuclei had 6\% lower extent $(p<0.01,64 \%$ $A \cup C$ ), and $0.2 \%$ lower fractal dimension ( $p<0.01,66 \%$ AUC), which indicated overall higher global shape irregularity and less intensively folded and wrinkled nuclear surfaces, respectively.

Overall, we showed that VPA robustly induced more elongated and less spherical nuclear shapes with higher mean curvature from day 3 . Towards day 7 , these nuclei demonstrated more prominent deformations characterized, for example, by a kidney-like form and blebbing (Figure 4C). At the same time, the decrease in curvedness and fractal dimension reflected nuclear surfaces with lower local border complexity that has also been suggested to be indicative of chromatin decondensation, reduced tumorigenesis, and neuroprotection (Metze et al., 2019). Combined with the size features, these 3D shape descriptors enable more accurate characterization of time-dependent morphological changes than their 2D counterparts or any single measure used individually.

Together, our findings described the dynamics of VPA-treated nuclear morphological profiles characterized by the increased sizes and progressively more irregular, complex shapes that can be attributed to altered histone modifications and chromatin decondensation. These observations represent a first step to studying time-dependent morphological effects of chromatin reorganization in the astrocyte-to-neuron reprogramming process and relating them to underlying molecular mechanisms. In future studies, this approach can be extended to label additional subnuclear components or organelles, such as nucleoli, chromosome territories, TADs, transcriptional condensates, and other compartments using, for example, Cell Painting assay(s) for high-content morphological profiling (Bray et al., 2016). While tracking the overall cellular phenotype, this extension will allow us to include many other features in the models and assess their variability, association with cellular and nuclear shape morphology, disease state, and treatment conditions. All of this will set the stage to evaluate the effects of VPA and other small molecules measured with different concentrations and temporal sampling. Correlating these phenotypical cell and nuclear profiles with data from other assays, such as Hi-C (LiebermanAiden et al., 2009), will likely provide useful insights into how altered functional properties of the genome are correlated with TAD structure, nuclear and cellular morphology, and can it be used for training machine learning models to more accurately predict treatment response in model systems and in humans (Kalinin et al., 2018c). 


\section{Methods}

\section{Sample preparation and image acquisition}

Primary human astrocyte cells were purchased from ScienCell (Human Astrocytes hippocampal, Catalog \#1830).

Day 0:

Replace media with 50\% growth media and 50\% N2 media (DMEM/F12 + 1X pen/strep, 1X N2 supplements).

Day 1:

Control samples (NHA): for $30 \mathrm{ml}$ of $\mathrm{N} 2$ media add $36 \mathrm{ul}$ DMSO

VPA-treated (1.5mM VPA): for $30 \mathrm{ml}$ of $\mathrm{N} 2$ media add 450ul VPA

Day 3:

Collect day 3 control and VPA samples:

- fix samples in 4\% PFA for $10 \mathrm{~min}$

- rinse $3 \times 5$ min in PBS

- store samples in PBS at $4 \mathrm{deg}$

Control samples (NHA): for $30 \mathrm{ml}$ of $\mathrm{N} 2$ media add 187.5ul DMSO

VPA-treated (1.5mM VPA): for $30 \mathrm{ml}$ of $\mathrm{N} 2$ media add 450ul VPA

Day 5 :

Collect day 5 control and VPA samples:

- fix samples in 4\% PFA for $10 \mathrm{~min}$

- rinse $3 \times 5$ min in PBS

- store samples in PBS at 4deg

Control samples (NHA): for $30 \mathrm{ml}$ of $\mathrm{N} 2$ media add $37.5 \mathrm{ul}$ DMSO

VPA-treated (1.5mM VPA): for $30 \mathrm{ml}$ of $\mathrm{N} 2$ media add 450ul VPA

\section{Day 7:}

Collect day 7 control and VPA samples:

- fix samples in 4\% PFA for $10 \mathrm{~min}$ 
- rinse $3 \times 5$ min in PBS

- $\quad$ store samples in PBS at 4 deg

Cells in both collections were labeled with DAPI (4',6-diamidino-2-phenylindole), a common stain for the nuclear DNA. 3D imaging employed a Zeiss LSM 710 laser scanning confocal microscope with a 63x PLAN/Apochromat 1.4NA DIC objective. Each original 3D volume was then re-sliced into a $1,024 \times 1,024 \times Z$ lattice $(Z=\{30,50\})$, where regional sub-volumes facilitate the alignment with the native tile size of the microscope. For every sub-volume, accompanying vendor metadata was extracted from the original data.

\section{Image pre-processing and segmentation}

To correct for imaging artifacts, including the effect of axial smearing on volumetric measures, we deconvolved the obtained images. Theoretical 3D point spread functions for each individual image volume were modeled using the Richards \& Wolf algorithm from the PSF Generator plugin for Fiji (Kirshner et al., 2013). We then used estimated point spread functions and imaging metadata to apply Lucy-Richardson deconvolution (10 iterations) to the original 3D image volumes using the DeconvolutionLab2 software (Sage et al., 2017). Deconvolution reduced axial smearing and improved segmentation and classification results (Supplementary Figure 1).

We performed the automatic 3D segmentation of nuclei from deconvolved images using Nuclear Segmentation algorithm from the Farsight toolkit (Al-Kofahi et al., 2010; Kalinin et al., 2018a). The main advantage of this tool is that it was created specifically to segment DAPI-stained nuclei in $2 \mathrm{D}$ or $3 \mathrm{D}$. Unlike other learning-based segmentation algorithms, it does not require a labeled training set. It demonstrated stable results in our previous project (Kalinin et al., 2018a) and on these data. The algorithm implements multiple steps, which include a graph-cut algorithm to binarize the sub-volumes, a multi-scale Laplacian of Gaussian filter to convert the nuclei to blob masks, fast clustering to delineate the nuclei, and nuclear contour refinement using graph-cuts with alpha-expansions (Al-Kofahi et al., 2010).

After segmentation of the DAPI channel sub-volumes, data were converted to 16-bit 3D TIFF files, each segmented nucleus was represented as a binary mask, and given a unique index value. Post-segmentation processing of nuclear masks included 3D hole filling and a filtering step that removed the objects if they span the edge of a tile, are connected to other objects. This quality control protocol filtered out most of the artifacts and remaining were removed by visual inspection. 


\section{D morphometry}

3D binary nuclear masks were then used for voxel-based feature extraction using scikit-image Python library (van der Walt et al., 2014), obtaining feature set $V$. For each binary mask we measured nuclear volume, bounding box and convex hull volumes, extent, solidity, lengths of principal axes, inertia tensor principal components, and diameter of the sphere with the same volume as the nucleus. In order to compare performance of $2 \mathrm{D}$ vs 3D features, we also extracted 2D features from maximum intensity projections of binary masks.

First, we model boundaries of nuclear 3D masks extracted from the microscopic images as genus zero two-dimensional manifolds that are embedded as triangulated surfaces in $\mathbb{R}^{3}$ (Kalinin et al., 2018b). 3D surface modeling uses an iterative Laplace-Beltrami eigen-projection and a topologypreserving boundary deformation algorithm (Shi et al., 2010). This algorithm performs robust reconstruction of the objects' surfaces from their segmented masks using an iterative mask filtering process. We used Mask2Mesh implementation of this algorithm from the MOCA framework (Shi et al., 2010) and then applied mesh simplification and subdivision to 40,000 triangles, following the shape analysis protocol in (Kalinin et al., 2018b). The next step included extraction of geometric characteristics of the 3D nuclear surfaces, e.g., mesh volume, surface area, curvedness, shape index, and fractal dimension, implemented in LONI Pipeline v7.0.3 (Dinov et al., 2009; Kalinin et al., 2018b). In order to provide a comprehensive morphological characterization, we expanded the list of six metrics previously reported in (Kalinin et al., 2018b). The additional surface features were extracted using trimesh library (Dawson-Haggerty et al., 2019) and included average mean curvature, convex hull and bounding primitive (box, oriented box, cylinder, sphere) volumes, convex hull surface area, inertia tensor eigenvalues, principal axes lengths. Surface-based extent and solidity were computed from trimesh-derived measures as the ratio of the object volume to the bounding box volume and the ratio of the object volume to the convex hull volume, correspondingly. Sphericity of the nucleus was computed as the ratio of the surface area of a sphere with the same volume as the given nucleus to the surface area of the nucleus: $\Psi=\frac{\pi^{\frac{1}{3}}(6 V)^{\frac{2}{3}}}{S A}$, where $V$ is the volume of the nucleus and $S A$ is the surface area of the nucleus (Wadell, 1935). All surface descriptors were combined into the feature set $S$.

Both volumetric and surface features we merged by inner join on the per-nucleus basis, filtering out those individual cells, for which feature extraction failed or voxel volume measure exceeded 
the empirically estimated threshold of 150000 voxels. Nuclei were also considered outliers and removed if any feature value computed over all cells was out of the $\left[P_{5}-1.5\left(P_{95}-P_{5}\right) ; P_{95}+\right.$ $\left.1.5\left(P_{95}-P_{5}\right)\right]$ range, where $P_{5}$ and $P_{95}$ are $5^{\text {th }}$ and $95^{\text {th }}$ percentiles, correspondingly. All extracted features were aggregated into per-nucleus feature vectors, from which we constructed a feature table per each day of treatment with the labels corresponding to phenotypic conditions (NHA and VPA).

\section{Feature and model selection, and analysis}

In order to handle multicollinear features, we performed hierarchical clustering on the Spearman rank-order correlations between all features and then used averaged SVM classification performance to select a threshold for defining feature clusters, while controlling for overfitting with 5-fold statistical cross-validation repeated from 5 different random seeds. From each cluster, we selected one feature according to the highest value for the $\chi^{2}$ statistic, while ignoring inertia tensor eigenvalues, as they were highly correlated with other features that are easier to directly interpret, such as volume or minimal principal axis length. This process yielded the final set $S_{7}$ consisting of the following 7 descriptors: surface-based median axis length, convex hull and bounding sphere volumes, sphericity, average mean curvature, shape index, and voxel-based solidity. t-SNE embedding was generated using scikit-learn library (Pedregosa et al., 2011) with PCA initialization, perplexity of 13 , and cosine distance as a metric. Clusters for each condition at every timepoint were identified using kernel density estimation in seaborn library (Waskom et al., 2020) with 0.75 threshold. It should be noted that cluster sizes and inter-cluster distances should be interpreted with care when using t-SNE (Wattenberg et al., 2016).

Following sets of features were used to compare volumetric and surface-based shape representations: $V_{2 d}, V, S, V_{\text {sub }}, S_{\text {sub }}, V+S, S_{7}$, where $V_{\text {sub }}$ and $S_{\text {sub }}$ were subsets of equivalent voxel and surface feature sets correspondingly (nuclear volume, convex hull and bounding box volumes, extent, solidity, inertia eigenvalues, and major axis length). We assessed classification performance of those classifiers from with scikit-learn library (Pedregosa et al., 2011) that provided feature weights as an output. These included Gaussian naïve Bayes, k-nearest neighbors, logistic regression, linear support vector machine (SVM), random forest, extremely randomized trees, AdaBoost and gradient boosting machine. They were trained using default hyperparameter values and evaluated at each timepoint using the Area under the Receiving Operator Characteristic. AUCs, confusion matrices, and feature ranking were averaged from 10 repetitions of the internal statistical 5-fold stratified cross-validation with different random seeds. 
We averaged the performance of each model over timepoints and over all feature sets. To compare feature sets, we averaged the AUCs over all models and days. Random subsampling of the prevalent class by the total number of per-fold training samples from an underrepresented class at each iteration of the cross-validation procedure was used to combat class imbalance. To rank features by their relative importance, we employed the permutation importance strategy that reflects the decrease in a model performance when a single feature value is randomly shuffled (Breiman, 2001). This breaks the relationship between the feature and the outcome, while being model agnostic and can be calculated many times with different permutations of the feature. We computed permutation importance on a held-out set on each cross-validation cycle, highlighting which features contribute the most to the generalization power of the trained model. Permutation feature importance was computed using the 'coef_' property of the trained SVM model at each timepoint.

Univariate statistical analysis of individual features was performed using SciPy package (Virtanen et al., 2020) with multiple testing correction using statsmodels (Seabold and Perktold, 2010). We reported each relative difference as the percentage change from the control group, along with a p-value obtained using two-sided Mann-Whitney $U$ test with Holm-Šidák multiple testing correction $(\alpha=0.05)$, along with the difference of means (DoM), and the common language effect size statistic that in the case of Mann-Whitney $U$ test is equivalent to the Area under the Receiving Operator Characteristic curve (AUC) (Mason and Graham, 2002).

For all analysis tasks we used Python 3.8.3 from the Anaconda distribution (Continuum Analytics and others, 2016), with numpy (van der Walt et al., 2011), pandas (McKinney, 2010), and iPython (Perez and Granger, 2007) packages. Image processing was done using scikit-image (van der Walt et al., 2014). Visualizations and charts were built with matplotlib (Hunter, 2007), seaborn (Waskom et al., 2020), trimesh (Dawson-Haggerty et al., 2019), and SOCRAT (Kalinin et al., 2017) libraries.

The documentation supporting the conclusions of this article together with the derived data, pipeline workflows, and underlying source code will be made publicly available online upon publication of the paper on the project webpage: SOCR 3D Cell Morphometry Project, https://socr.umich.edu/projects/3d-cell-morphometry. 


\section{Acknowledgements}

The authors thank Ari Allyn-Feuer, Samuel Handelman, and Gilbert Omenn for thoughtful discussions and helpful suggestions. We thank colleagues at the Laboratory of Neuro Imaging (LONI), at the Keck School of Medicine, University of Southern California, for providing technical support for the LONI Pipeline environment. This work was partially supported by the National Science Foundation grants 1916425, 1734853, 1636840, 1416953, 0716055, 1023115; the National Institutes of Health grants P20 NR015331, P30 DK089503, UL1TR002240, R01CA233487, R01MH121079. Xin Rong of the University of Michigan donated NVIDIA Titan X GPU used for this research, and the NVIDIA Corporation donated TITAN Xp GPU used to execute the computationally intensive protocol. 


\section{References}

Al-Kofahi, Y, Lassoued, W, Lee, W, and Roysam, B (2010). Improved automatic detection and segmentation of cell nuclei in histopathology images. IEEE Transactions on Biomedical Engineering 57, 841-852.

Amamoto, R, and Arlotta, P (2014). Development-inspired reprogramming of the mammalian central nervous system. Science 343, 1239882.

Bray, M-A, Singh, S, Han, H, Davis, CT, Borgeson, B, Hartland, C, Kost-Alimova, M, Gustafsdottir, SM, Gibson, CC, and Carpenter, AE (2016). Cell Painting, a high-content imagebased assay for morphological profiling using multiplexed fluorescent dyes. Nature Protocols $11,1757-1774$.

Breiman, L (2001). Random Forests. Machine Learning 45, 5-32.

Chen, H, Chen, J, Muir, LA, Ronquist, S, Meixner, W, Ljungman, M, Ried, T, Smale, S, and Rajapakse, I (2015). Functional organization of the human 4D Nucleome. Proc Natl Acad Sci USA 112, 8002-8007.

Cheng, L, Gao, L, Guan, W, Mao, J, Hu, W, Quu, B, Zhao, J, Yu, Y, and Pei, G (2015). Direct conversion of astrocytes into neuronal cells by drug cocktail. Cell Research 25, 1269.

Choi, H-J, and Choi, H-K (2007). Grading of renal cell carcinoma by 3D morphological analysis of cell nuclei. Computers in Biology and Medicine 37, 1334-1341.

Continuum Analytics and others (2016). Anaconda software distribution. Computer Software Vers, 2-2.

Cortes, C, and Vapnik, V (1995). Support-vector networks. Mach Learn 20, 273-297.

Cremer, T, Cremer, M, Hübner, B, Strickfaden, H, Smeets, D, Popken, J, Sterr, M, Markaki, Y, Rippe, K, and Cremer, C (2015). The 4D nucleome: Evidence for a dynamic nuclear landscape based on co-aligned active and inactive nuclear compartments. FEBS Letters 589, 2931-2943.

Dawson-Haggerty et al. (2019). trimesh.

Depeursinge, A, Foncubierta-Rodriguez, A, Van De Ville, D, and Müller, H (2014). Threedimensional solid texture analysis in biomedical imaging: Review and opportunities. Medical Image Analysis 18, 176-196.

Dinov, I, Van Horn, J, Lozev, K, Magsipoc, R, Petrosyan, P, Liu, Z, MacKenzie-Graha, A, Eggert, P, Parker, DS, and Toga, AW (2009). Efficient, distributed and interactive neuroimaging data analysis using the LONI pipeline. Frontiers in Neuroinformatics 3, 22.

Dufour, AC, Liu, T-Y, Ducroz, C, Tournemenne, R, Cummings, B, Thibeaux, R, Guillen, N, Hero, AO, and Olivo-Marin, J-C (2015). Signal processing challenges in quantitative 3-d cell morphology: More than meets the eye. IEEE Signal Processing Magazine 32, 30-40.

Eckschlager, T, Plch, J, Stiborova, M, and Hrabeta, J (2017). Histone Deacetylase Inhibitors as Anticancer Drugs. IJMS 18, 1414. 
Felisbino, MB, Costa, TA da, Gatti, MSV, and Mello, MLS (2016). Differential Response of Human Hepatocyte Chromatin to HDAC Inhibitors as a Function of Microenvironmental Glucose Level. Journal of Cellular Physiology 231, 2257-2265.

Felisbino, MB, Gatti, MSV, and Mello, MLS (2014). Changes in Chromatin Structure in NIH 3T3 Cells Induced by Valproic Acid and Trichostatin A. Journal of Cellular Biochemistry 115, 19371947.

Felisbino, MB, Tamashiro, WMSC, and Mello, MLS (2011). Chromatin Remodeling, Cell Proliferation and Cell Death in Valproic Acid-Treated HeLa Cells. PLoS ONE 6, e29144.

Ganai, SA, Malli Kalladi, S, and Mahadevan, V (2015). HDAC inhibition through valproic acid modulates the methylation profiles in human embryonic kidney cells. Journal of Biomolecular Structure and Dynamics 33, 1185-1197.

Gao, L et al. (2017). Direct generation of human neuronal cells from adult astrocytes by small molecules. Stem Cell Reports 8, 538-547.

Göttlicher, M et al. (2001). Valproic acid defines a novel class of HDAC inhibitors inducing differentiation of transformed cells. The EMBO Journal 20, 6969-6978.

Higgins, GA, Allyn-Feuer, A, Georgoff, P, Nikolian, V, Alam, HB, and Athey, BD (2017a). Mining the topography and dynamics of the 4D Nucleome to identify novel CNS drug pathways. Methods 123, 102-118.

Higgins, GA, Allyn-Feuer, A, Handelman, S, Sadee, W, and Athey, BD (2015). The epigenome, 4D nucleome and next-generation neuropsychiatric pharmacogenomics. Pharmacogenomics $16,1649-1669$.

Higgins, GA, Georgoff, P, Nikolian, V, Allyn-Feuer, A, Pauls, B, Higgins, R, Athey, BD, and Alam, HE (2017b). Network Reconstruction Reveals that Valproic Acid Activates Neurogenic Transcriptional Programs in Adult Brain Following Traumatic Injury. Pharm Res 34, 1658-1672.

Huangfu, D, Maehr, R, Guo, W, Eijkelenboom, A, Snitow, M, Chen, AE, and Melton, DA (2008). Induction of pluripotent stem cells by defined factors is greatly improved by small-molecule compounds. Nature Biotechnology 26, 795-797.

Hunter, JD (2007). Matplotlib: A 2D Graphics Environment. Computing in Science Engineering 9, 90-95.

Kalinin, AA et al. (2018a). 3D Cell Nuclear Morphology: Microscopy Imaging Dataset and VoxelBased Morphometry Classification Results. In: Proceedings of the IEEE Conference on Computer Vision and Pattern Recognition Workshops, 2272-2280.

Kalinin, AA et al. (2018b). 3D Shape Modeling for Cell Nuclear Morphological Analysis and Classification. Scientific Reports 8, 13658.

Kalinin, AA, Higgins, GA, Reamaroon, N, Soroushmehr, S, Allyn-Feuer, A, Dinov, ID, Najarian, $\mathrm{K}$, and Athey, BD (2018c). Deep learning in pharmacogenomics: from gene regulation to patient stratification. Pharmacogenomics 19, 629-650. 
Kalinin, AA, Palanimalai, S, and Dinov, ID (2017). SOCRAT Platform Design: A Web Architecture for Interactive Visual Analytics Applications. In: Proceedings of the 2nd Workshop on Human-In-the-Loop Data Analytics, New York, NY, USA: ACM, 8:1-8:6.

Kirshner, H, Aguet, F, Sage, D, and Unser, M (2013). 3-D PSF fitting for fluorescence microscopy: implementation and localization application. Journal of Microscopy 249, 13-25.

Koenderink, JJ, and Van Doorn, AJ (1992). Surface shape and curvature scales. Image and Vision Computing 10, 557-564.

Kortenhorst, MSQ, Isharwal, S, van Diest, PJ, Chowdhury, WH, Marlow, C, Carducci, MA, Rodriguez, R, and Veltri, RW (2009). Valproic acid causes dose- and time-dependent changes in nuclear structure in prostate cancer cells in vitro and in vivo. Mol Cancer Ther 8, 802-808.

Lieberman-Aiden, E et al. (2009). Comprehensive Mapping of Long-Range Interactions Reveals Folding Principles of the Human Genome. Science 326, 289-293.

van der Maaten, L, and Hinton, G (2008). Visualizing Data using t-SNE. Journal of Machine Learning Research 9, 2579-2605.

Marchion, DC, Bicaku, E, Daud, Al, Sullivan, DM, and Munster, PN (2005). Valproic Acid Alters Chromatin Structure by Regulation of Chromatin Modulation Proteins. Cancer Res 65, 38153822.

Mason, SJ, and Graham, NE (2002). Areas beneath the relative operating characteristics (ROC) and relative operating levels (ROL) curves: Statistical significance and interpretation. Quarterly Journal of the Royal Meteorological Society 128, 2145-2166.

McKinney, W (2010). Data Structures for Statistical Computing in Python. Proceedings of the 9th Python in Science Conference, 56-61.

Medyukhina, A, Blickensdorf, M, Cseresnyés, Z, Ruef, N, Stein, JV, and Figge, MT (2020). Dynamic spherical harmonics approach for shape classification of migrating cells. Sci Rep 10, 6072.

Metze, K, Adam, R, and Florindo, JB (2019). The fractal dimension of chromatin - a potential molecular marker for carcinogenesis, tumor progression and prognosis. Expert Review of Molecular Diagnostics 19, 299-312.

Meyer, MG, Fauver, M, Rahn, JR, Neumann, T, Patten, FW, Seibel, EJ, and Nelson, AC (2009). Automated cell analysis in 2D and 3D: A comparative study. Pattern Recognition 42, 141-146.

Olson, RS, Cava, WL, Mustahsan, Z, Varik, A, and Moore, JH (2018). Data-driven advice for applying machine learning to bioinformatics problems. In: Biocomputing 2018, Kohala Coast, Hawaii, USA: WORLD SCIENTIFIC, 192-203.

Pedregosa, F et al. (2011). Scikit-learn: Machine Learning in Python. Journal of Machine Learning Research 12, 2825-2830.

Perez, F, and Granger, BE (2007). IPython: A System for Interactive Scientific Computing. Computing in Science Engineering 9, 21-29. 
Pickell, Z, Williams, AM, Alam, HB, and Hsu, CH (2020). Histone Deacetylase Inhibitors: A Novel Strategy for Neuroprotection and Cardioprotection Following Ischemia/Reperfusion Injury. Journal of the American Heart Association 9, e016349.

Qin, $\mathrm{H}$, Zhao, A, and $\mathrm{Fu}, \mathrm{X}$ (2017). Small molecules for reprogramming and transdifferentiation. Cell Mol Life Sci 74, 3553-3575.

Sage, D, Donati, L, Soulez, F, Fortun, D, Schmit, G, Seitz, A, Guiet, R, Vonesch, C, and Unser, M (2017). DeconvolutionLab2: An open-source software for deconvolution microscopy. Methods $115,28-41$.

Sasai, K, Akagi, T, Aoyanagi, E, Tabu, K, Kaneko, S, and Tanaka, S (2007). O6-methylguanineDNA methyltransferase is downregulated in transformed astrocyte cells: implications for antiglioma therapies. Mol Cancer 6, 36.

Seabold, S, and Perktold, J (2010). statsmodels: Econometric and statistical modeling with python. In: 9th Python in Science Conference.

Shi, Y, Lai, R, Morra, JH, Dinov, I, Thompson, PM, and Toga, AW (2010). Robust surface reconstruction via Laplace-Beltrami eigen-projection and boundary deformation. IEEE Transactions on Medical Imaging 29, 2009-2022.

Stephens, AD, Banigan, EJ, Adam, SA, Goldman, RD, and Marko, JF (2017). Chromatin and lamin A determine two different mechanical response regimes of the cell nucleus. MBoC 28 , 1984-1996.

Stephens, AD, Banigan, EJ, and Marko, JF (2019). Chromatin's physical properties shape the nucleus and its functions. Current Opinion in Cell Biology 58, 76-84.

Stephens, AD, Liu, PZ, Banigan, EJ, Almassalha, LM, Backman, V, Adam, SA, Goldman, RD, and Marko, JF (2018). Chromatin histone modifications and rigidity affect nuclear morphology independent of lamins. MBoC 29, 220-233.

Tsagkrasoulis, D, Hysi, P, Spector, T, and Montana, G (2017). Heritability maps of human face morphology through large-scale automated three-dimensional phenotyping. Sci Rep 7, 45885.

Uhler, C, and Shivashankar, GV (2018). Nuclear Mechanopathology and Cancer Diagnosis. Trends in Cancer 4, 320-331.

Virtanen, P et al. (2020). SciPy 1.0: fundamental algorithms for scientific computing in Python. Nature Methods 17, 261-272.

Wadell, $H$ (1935). Volume, shape, and roundness of quartz particles. The Journal of Geology 43, 250-280.

van der Walt, S, Colbert, SC, and Varoquaux, G (2011). The NumPy Array: A Structure for Efficient Numerical Computation. Computing in Science Engineering 13, 22-30.

van der Walt, S, Schönberger, JL, Nunez-Iglesias, J, Boulogne, F, Warner, JD, Yager, N, Gouillart, E, and Yu, T (2014). scikit-image: image processing in Python. PeerJ 2, e453. 
Waskom, M et al. (2020). mwaskom/seaborn: v0.10.1 (April 2020), Zenodo.

Wattenberg, M, Viégas, F, and Johnson, I (2016). How to Use t-SNE Effectively. Distill 1, e2.

Xu, D, Cui, J, Bansal, R, Hao, X, Liu, J, Chen, W, and Peterson, BS (2009). The ellipsoidal area ratio: an alternative anisotropy index for diffusion tensor imaging. Magn Reson Imaging 27, 311-323.

Yang, X-J, and Seto, E (2007). HATs and HDACs: from structure, function and regulation to novel strategies for therapy and prevention. Oncogene 26, 5310-5318.

Yin, J-C et al. (2019). Chemical Conversion of Human Fetal Astrocytes into Neurons through Modulation of Multiple Signaling Pathways. Stem Cell Reports 12, 488-501.

Zhang, L et al. (2015). Small molecules efficiently reprogram human astroglial cells into functional neurons. Cell Stem Cell 17, 735-747. 

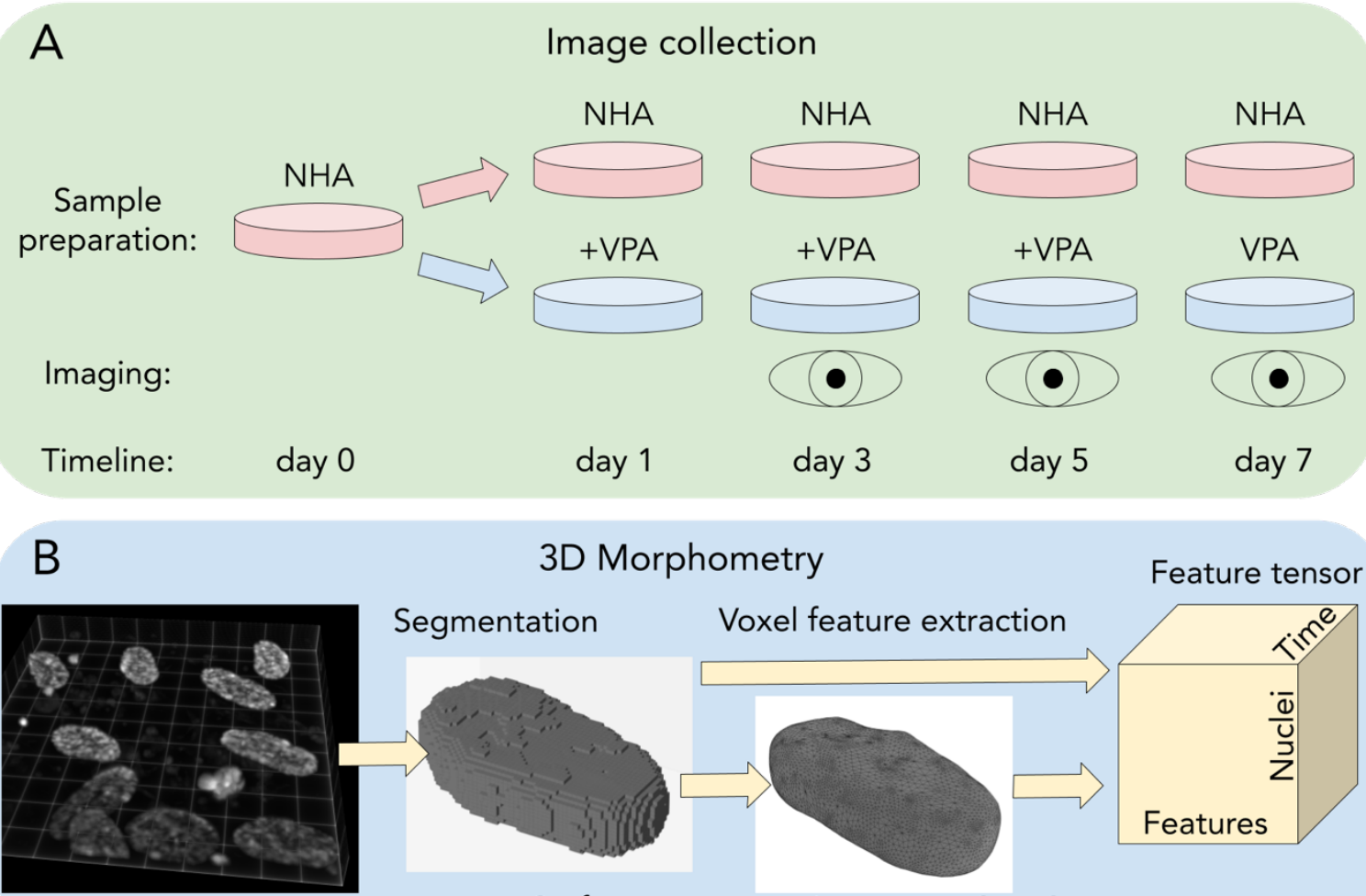

3D Morphometry

Feature tensor

C

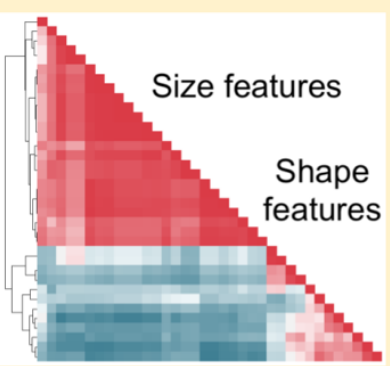

Feature selection
Analysis and interpretation

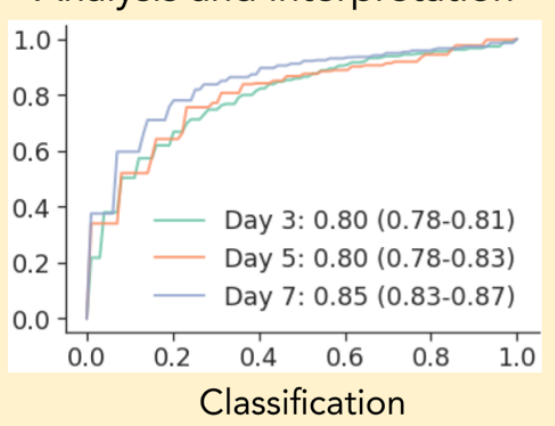

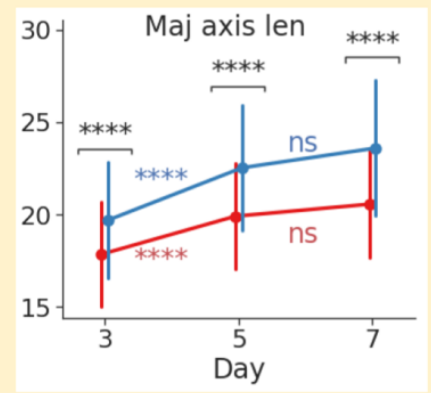

Statistical testing

Figure 1. A schematic overview of the experiment, data collection and analysis. (A) sample preparation, treatment, and imaging. (B) 3D nuclear segmentation, shape modeling, and feature extraction. (C) feature selection, univariate statistical and machine learning analysis. 
A

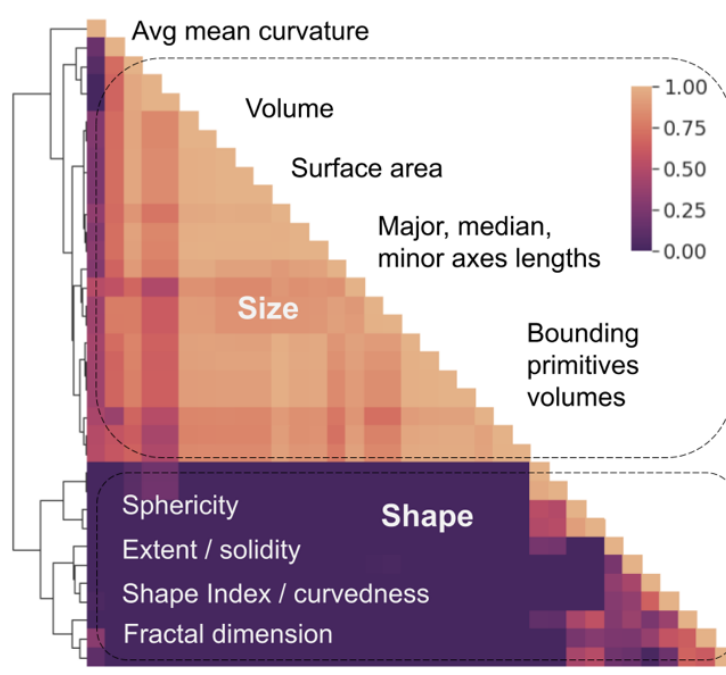

C

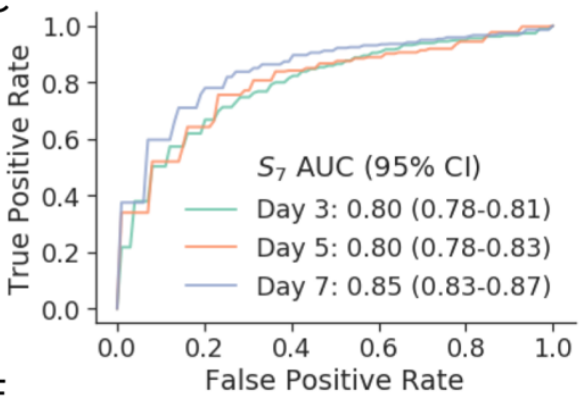

B
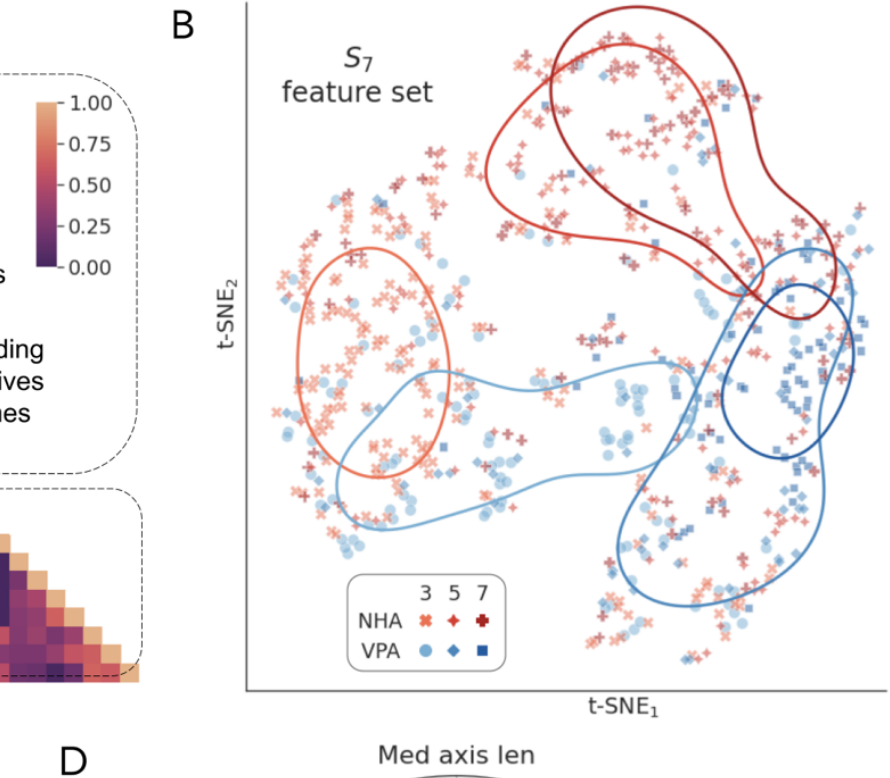
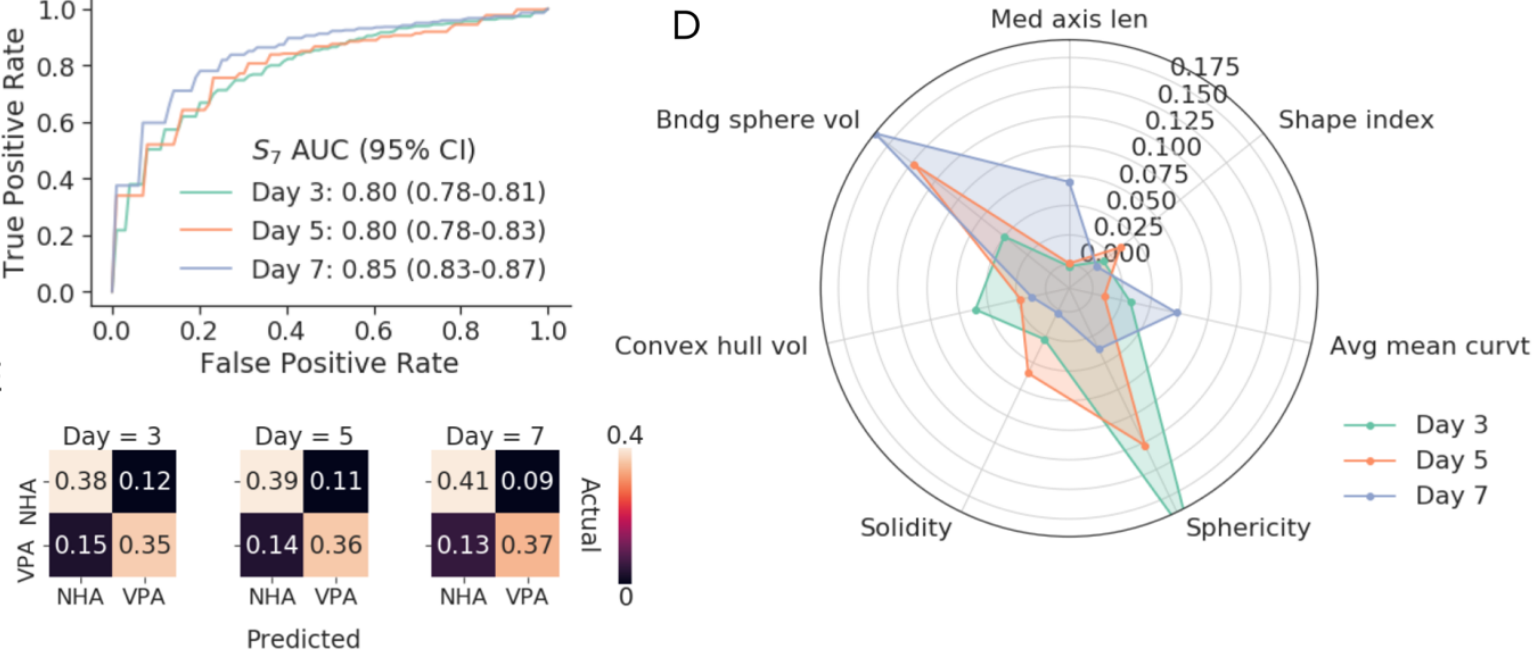

Figure 2. Morphological classification performance. (A) hierarchical clustering of the Pearson correlations among all voxel and surface features $(V+S)$, showing representative size and shape descriptors. (B) 2D t-Distributed stochastic neighbor embedding (t-SNE) of the selected feature space, showing corresponding conditions (HNA or VPA) at every timepoint (day 3,5 , or 7 ). The lines denote clusters identified by kernel density estimation. (C) ROC curves for the SVM classifier with $S_{7}$ features on days 3, 5, and 7. (D) average normalized confusion matrices for the SVM classifier on the $S_{7}$ features; (E) SVM-estimated permutation importance of $S_{7}$ features for distinguishing nuclear morphologies on each day. 
A

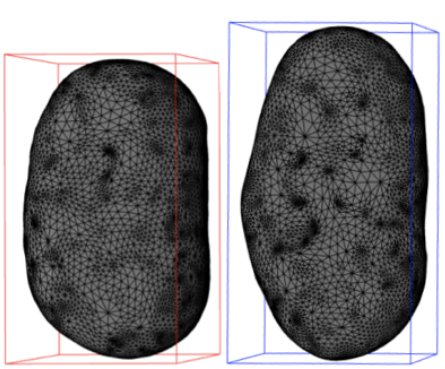

day 3

B
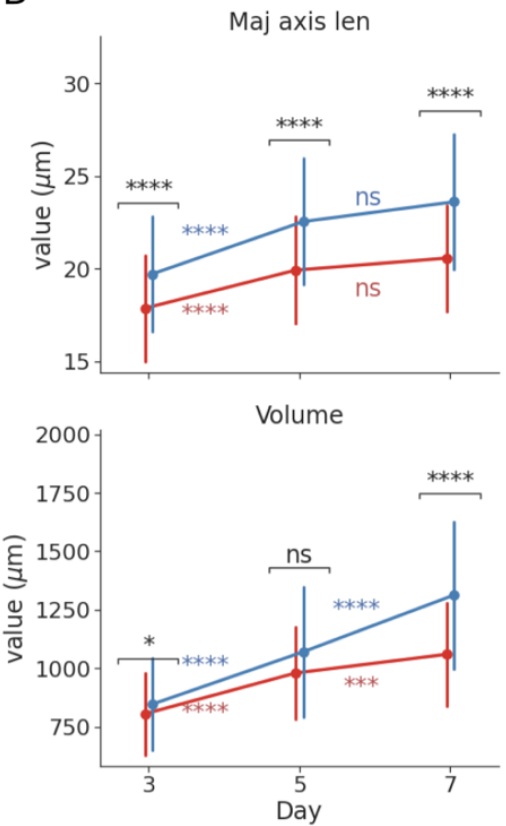

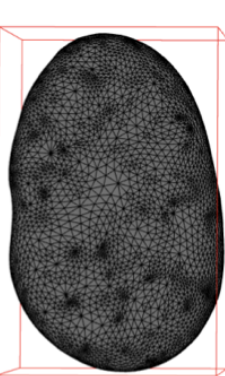

day 5

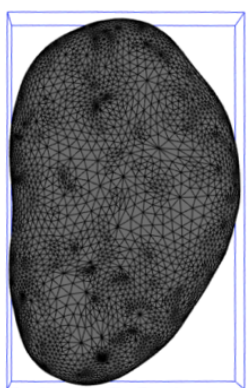

Med axis len

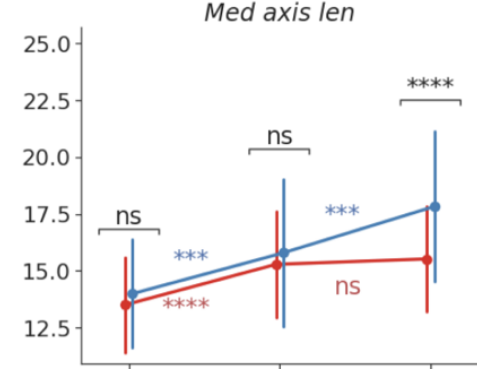

Convex hull volume

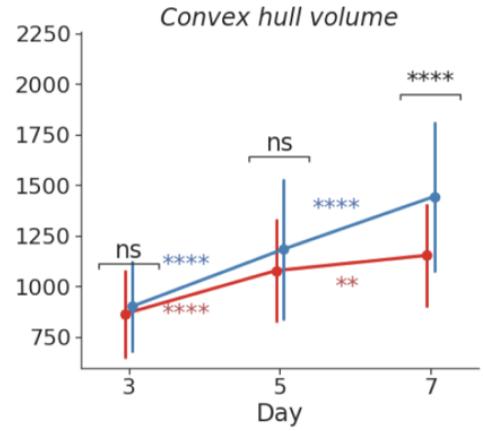

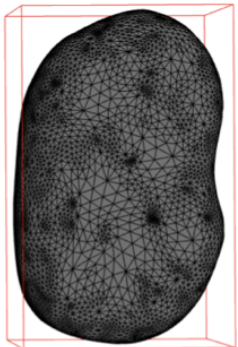

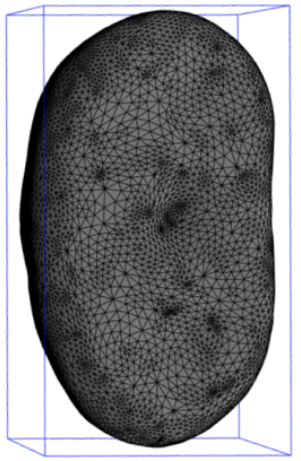

day 7

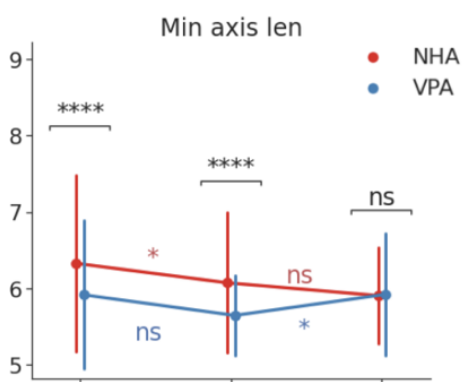

Bndg sphere volume

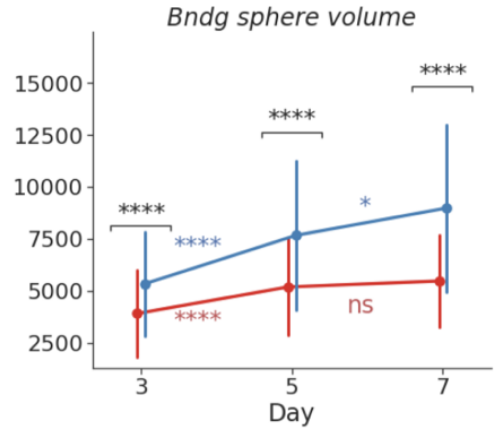

Figure 3. Visualization and univariate statistical analysis of size changes under VPA treatment. (A) Reconstructed surfaces of representative NHA and VPA nuclei on days 3, 5, and 7. (B) Timedependent changes in morphometric measures of nuclear sizes (points show mean; error bars show SD; $* p<0.05, * * p<0.01, * * * p<0.001, * * * * p<0.0001)$. 
A

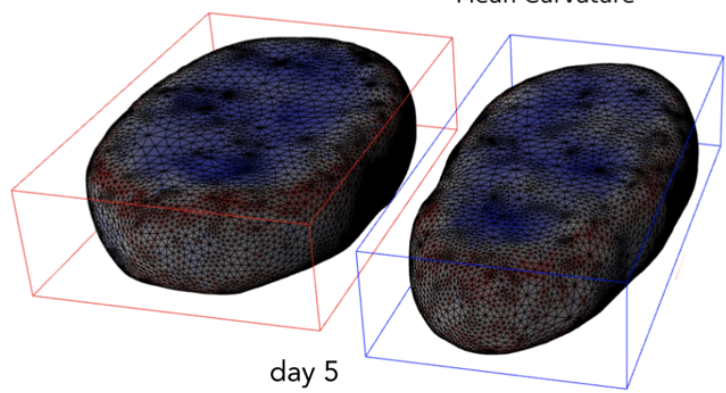

B

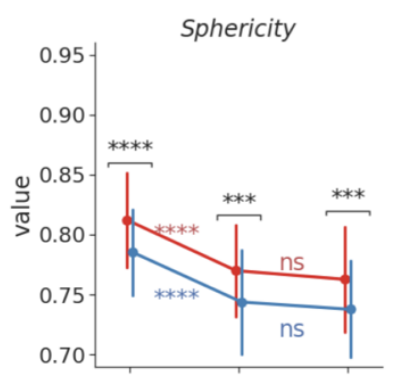

Shape index
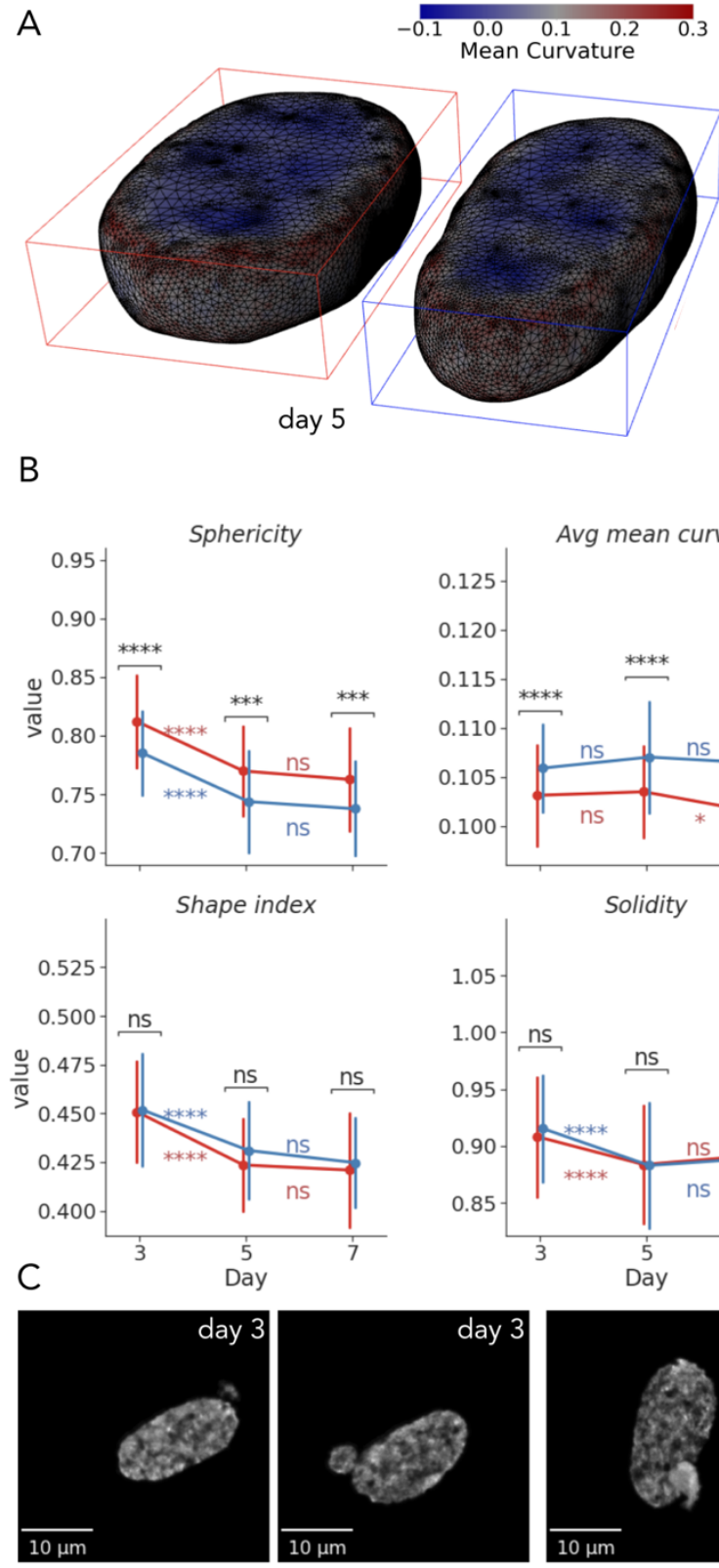

Mean Curvature

Solidity

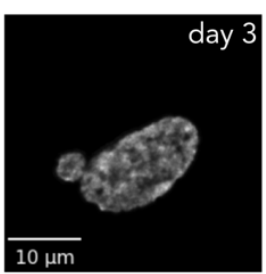

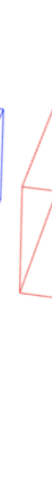

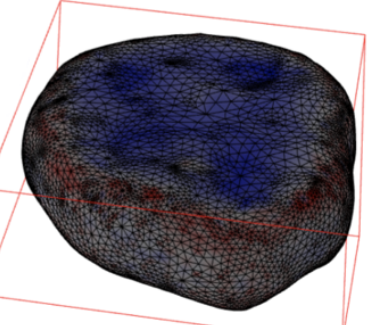

day 7

- NHA
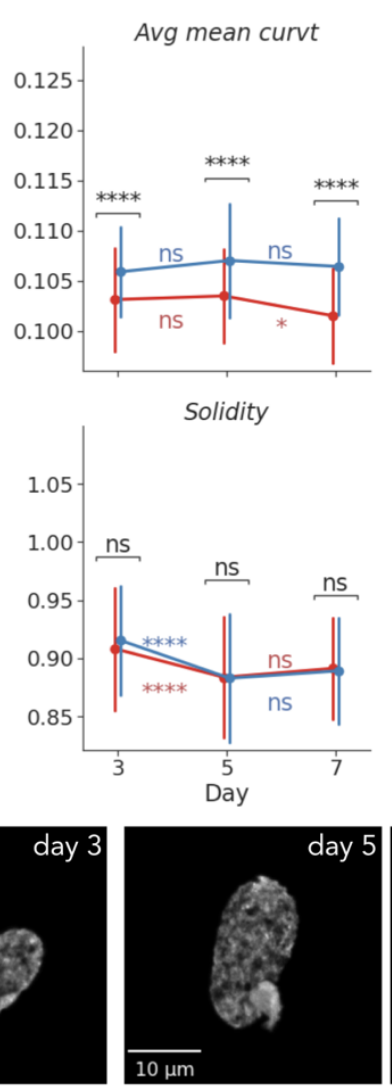

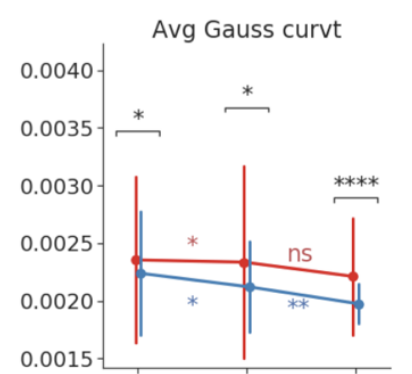

Extent

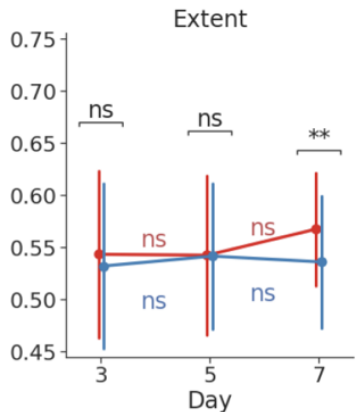

day 5

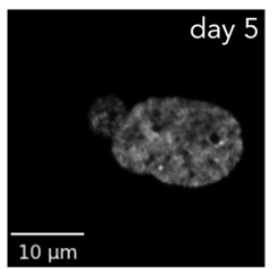

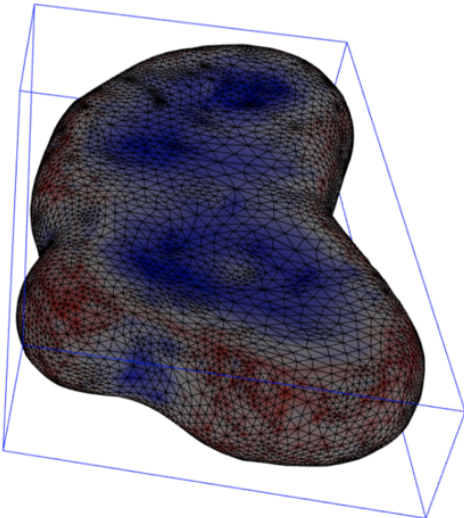

Curvedness

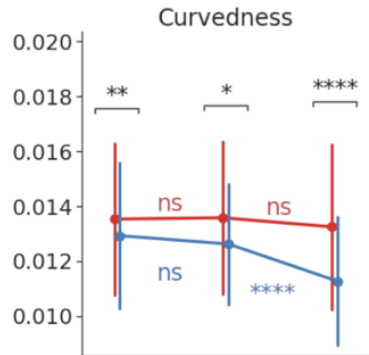

Fractal dim
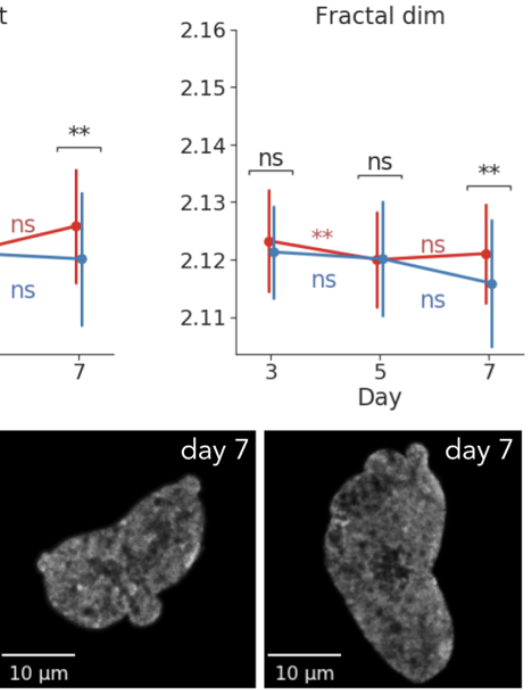

Figure 4. Visualization and univariate statistical analysis of shape changes under VPA treatment. (A) Reconstructed surfaces of a representative NHA and VPA nuclei on days 5 and 7, annotated with per-vertex mean curvature. (B) Time-dependent changes in morphometric measures of nuclear shapes (points show mean; error bars show SD; ${ }^{*} p<0.05,{ }^{* *} p<0.01,{ }^{* * *} p<0.001$, $* * * * p<0.0001)$. (C) $X Y$ maximum intensity projections of VPA-treated nuclei with irregular shapes and blebbing. 